\title{
New results of exponential synchronization of complex network with time-varying delays
}

\author{
Yiping Luo ${ }^{1}$, Zhaoming Ling ${ }^{1 *}$ (D) Zifeng Cheng ${ }^{1}$ and Bifeng Zhou ${ }^{1}$
}

\author{
"Correspondence: \\ Izmwz@foxmail.com \\ ${ }^{1}$ Hunan Institute of Engineering, \\ Xiangtan, China
}

\begin{abstract}
In this study, we elucidated the exponential synchronization of a complex network system with time-varying delay. Then the exponential synchronization control of several types of complex network systems with time-varying delay under no requirements of delay derivable were explored. The dynamic behavior of a system node shows time-varying delays. Thus, to derive suitable conditions for the exponential synchronization of different complex network systems, we designed a linear feedback controller for linear coupling functions, using the Lyapunov stability theory, Razumikhin theorem, and Newton-Leibniz formula. The exponential damping rates for the exponential synchronization of different complex network systems were then estimated. Finally, we validated our conclusions through a numerical simulation.
\end{abstract}

Keywords: Exponential synchronization; Complex network; Time-varying delay; Razumikhin theorem

\section{Springer}

\section{Introduction}

Many problems in nature can be descrobed by complex network models, such as the World Wide Web (WWW), food chains, traffic networks, and social networks. Thus, complex networks have attracted widespread interest.

Synchronization is an important dynamic characteristic of a complex network. Many phenomena in nature are realized by the synchronization of complex networks. For example, Strogatz discovered the synchronized contractions of cardiac muscle cells [1]. Steinmetz et al. discovered that the attention selection modes of human beings and primates are closely related with the synchronous rates of their neurons [2]. Furthermore, synchronization technologies have been widely applied in practical life. Millerioux et al. achieved chaos secure transmission from the master-slave system [3]. Kunbert et al. suggested solving problems encountered in image processing through the use of synchronously generated autowaves [4]. Synchronization control has received considerable attention in the control field, and many control technologies have been introduced into the synchronization of networks. Adaptive, pulse, intermittent, and pinning controls have been used for the synchronous control of complex networks and thereby contributed to many outstanding works [5-8].

(c) The Author(s) 2019. This article is distributed under the terms of the Creative Commons Attribution 4.0 International License (http://creativecommons.org/licenses/by/4.0/), which permits unrestricted use, distribution, and reproduction in any medium, provided you give appropriate credit to the original author(s) and the source, provide a link to the Creative Commons license, and indicate if changes were made. 
Information transition in a network is often accompanied by time delay, which is a major cause of system instability. Therefore, studying the stability of a time-delay system has become a critical topic in control theory. Given that the time-delay system is an infinitedimensional system, studying it is difficult, and no general research method for its study is currently available. Moreover, different problems require different control technologies. This condition often results in inconsistent research conclusions [9, 10]. Nevertheless, research on methods for the synchronous control of complex networks with time delay has achieved considerable progress [11-16].

Given that time delay always changes, most studies mainly focus on the synchronization of complex network model with time-varying delay. For research on time-varying delay there are two requirements. First, time-varying delay should have boundaries [1719]. Second, the derivative of a time-varying delay should satisfy only one condition. It generally requires the derivative of a time-varying delay. This derivative should be smaller than 1 [20-24] or should be localized in one boundary [25-27]. Meanwhile, the derivation of a rapidly changing time-varying delay may fail, and thus these methods are ineffective. Inspired by Ref. [9], we propose a novel method to address the problems encountered in the synchronous control of a complex network with time-varying delay. In this method, a derivable time-varying delay is unnecessary, and stability conditions for the exponential synchronization of systems are concluded by the Razumikhin theorem. This study has some innovation points:

(1) The time-varying delay function is removed as a derivable in the study of synchronous control of common time-delay complex systems. The synchronous control problem in complex systems with time-varying delay is studied by using the Razumikhin theorem and the Newton-Leibniz formula.

(2) Several types of complex network systems with time-varying delay are nonlinear functions and contain time-varying delays, and the conditions for exponential synchronization are obtained on the basis of the hypothesis that the nonlinear function satisfies the Lipschitz function, whether or not the coupling function between the network nodes is linear. These conditions satisfy a linear matrix inequality and are easy to determine. The upper boundary of time-varying delay can easily be calculated with the MATLAB tool box.

Notations All the notations used in this paper are standard. For $x \in \mathbb{R}^{n}$, let $\|x\|$ denote the Euclidean vector norm, i.e., $\|x\|=\sqrt{\left(x^{T} x\right)}$. For $A \in \mathbb{R}^{N \times N}$, let $\|P\|$ indicate the norm of $P$ induced by the Euclidean vector norm, i.e., $\|P\|=\sqrt{\eta_{\max }\left(P^{T} P\right)}$, where $\eta_{\max }\left(P^{T} P\right)$ is the maximum eigenvalue of $P^{T} P, I$ denotes the identity matrix with appropriate dimensions, $\mathbb{R}^{m \times n}$ denotes the set of all $m \times n$ real matrices, and $\mathbb{R}^{n}$ is the $n$-dimensional Euclidean space. For symmetric matrices $A$ and $B$, the notation $A>B(A \geq B)$ means that the matrix $A-B$ is positive definite (nonnegative); $\operatorname{diag}\{\ldots\}$ is used to denote the block diagonal matrix; the superscript “ $T$ ” represents the transpose; let $A \otimes B$ be the Kronecker product of two matrices $A$ and $B$. 


\section{Preliminaries}

We consider a general time-varying delay complex dynamic network consisting of $N$ dynamic nodes with linear couplings, which is described by

$$
\begin{aligned}
& \dot{x}_{i}=f\left(x_{i}(t), x_{i}(t-\tau(t))\right)+c_{1} \sum_{j=1}^{N} a_{i j} \Gamma_{1} x_{j}(t)+c_{2} \sum_{j=1}^{N} b_{i j} \Gamma_{2} x_{j}(t-\tau(t)), \\
& x_{i}(t)=\phi(t), \quad t \in[-\tau, 0] .
\end{aligned}
$$

$x_{i}=\left(x_{i 1}(t), x_{i 2}(t), \ldots, x_{i n}(t)\right)^{T} \in \mathbb{R}^{n}$ is the state vector of the $i$ th leader, $f: \mathbb{R}^{n} \times \mathbb{R}^{n} \rightarrow$ $\mathbb{R}^{n}$ is a continuously differential nonlinear vector function, $c_{1}>0$ and $c_{2}>0$ are the non-delay and time-varying delay coupling strengths, $\Gamma_{1}=\operatorname{diag}\left(\gamma_{1}^{1}, \gamma_{1}^{2}, \ldots, \gamma_{1}^{n}\right)$ and $\Gamma_{2}=$ $\operatorname{diag}\left(\gamma_{2}^{1}, \gamma_{2}^{2}, \ldots, \gamma_{2}^{n}\right)$ are positive definite diagonal matrices, which represent the inner connection matrices between each pair of nodes; Meanwhile, $A=\left(a_{i j}\right) \in \mathbb{R}^{N \times N}, B=\left(b_{i j}\right) \in$ $\mathbb{R}^{N \times N}$ are the non-delay and time-varying delay weight matrices. The entries are defined as follows: if is a link is present from the node $i$ to the node $j(i \neq j)$, then $a_{i j} \neq 0, b_{i j} \neq 0$; otherwise $a_{i j}=0, b_{i j}=0$, and the diagonal elements of matrices $A$ and $B$ are defined as $a_{i i}=-\sum_{j=1, j \neq i}^{N} a_{i j}, b_{i i}=-\sum_{j=1, j \neq i}^{N} b_{i j}$. Given that the networks in this paper are direct, $a_{i j} \neq a_{j i}$ and $b_{i j} \neq b_{j i}(i, j=1,2, \ldots, N)$. The coupling time-varying delay $\tau(t)$ is a bound function and a positive constant $\tau$ satisfying $0 \leq \tau(t) \leq \tau, \forall t>0, u_{i} \in \mathbb{R}^{N \times N}$ is the control input of node $i$. In this paper, we aim to design a suitable controller

$$
u_{i}(t)=k_{i}\left(x_{i}(t)-s(t)\right)
$$

where $k_{i}$ is the gain of the $i$ th node.

Let $\tilde{f}(x(t), x(t-\tau(t)))=f(x(t), x(t-\tau(t)))-f(s(t), s(t-\tau(t)))$, and $K=\operatorname{diag}\left(k_{1}, k_{2}, \ldots, k_{n}\right)$.

We use the Newton-Leibniz formula

$$
x(t-\tau(t))=x(t)-\int_{t-\tau(t)}^{t} \dot{x}(s) d s .
$$

Equation (1) is converted to

$$
\begin{aligned}
\dot{x}(t)= & f(x(t), x(t-\tau(t)))+c_{1} A \otimes \Gamma_{1} x(t)+c_{2} B \otimes \Gamma_{2} x(t) \\
& -c_{2} B \otimes \Gamma_{2} \int_{t-\tau(t)}^{t}\left[f(x(v), x(v-\tau(v)))+c_{1} A \otimes \Gamma_{1} x(t)\right] d v \\
& -c_{2} B \otimes \Gamma_{2} \int_{t-\tau(t)}^{t} c_{2} B \otimes \Gamma_{2} x(v-\tau(v)) d v, \\
x(t)= & \psi(t), \quad t \in[-2 \tau, 0] .
\end{aligned}
$$

The set $s=\left\{x_{1}^{T}(t), x_{2}^{T}(t), \ldots, x_{n}^{T}(t) \in \mathbb{R}^{n} \mid x_{i}(t)=s(t), i \in \mathbb{N}\right\}$ is defined as synchronization manifold, where $s(t) \in \mathbb{R}^{n}$ satisfies

$$
\dot{s}(t)=f(s(t), s(t-\tau(t))) .
$$

The synchronization error vector is defined as

$$
e_{i}(t)=\left(x_{i}(t)-s(t), \quad e_{i}(t-\tau(t))=x_{i}(t-\tau(t))-s(t-\tau(t)) \quad(i=1, \ldots, N) .\right.
$$


Through Eqs. (4) and (5), we can obtain the following error system:

$$
\begin{aligned}
\dot{e}(t)= & \tilde{f}(x(t), x(t-\tau(t)))+c_{1} A \otimes \Gamma_{1} e(t)+c_{2} B \otimes \Gamma_{2} e(t) \\
& -c_{2} B \otimes \Gamma_{2} \int_{t-\tau(t)}^{t} \tilde{f}(x(v), x(v-\tau(v)))+c_{1} A \otimes \Gamma_{1} e(t) d v \\
& -c_{2} B \otimes \Gamma_{2} \int_{t-\tau(t)}^{t} c_{2} B \otimes \Gamma_{2} e(v-\tau(v)) d v .
\end{aligned}
$$

To achieve the synchronization of system (1), we add controller (2) to the error system and we have

$$
\begin{aligned}
& \dot{e}(t)=\tilde{f}(x(t), x(t-\tau(t)))+c_{1} A \otimes \Gamma_{1} e(t)+c_{2} B \otimes \Gamma_{2} e(t) \\
&-c_{2} B \otimes \Gamma_{2} \int_{t-\tau(t)}^{t} \tilde{f}(x(v), x(v-\tau(v)))+c_{1} A \otimes \Gamma_{1} e(t) d v \\
&-c_{2} B \otimes \Gamma_{2} \int_{t-\tau(t)}^{t} c_{2} B \otimes \Gamma_{2} e(v-\tau(v)) d v+K e(t) .
\end{aligned}
$$

Therefore, if the error system (6) is exponentially stable, then the complex network (1) is exponentially synchronized.

The following assumptions and lemmas are needed for the derivation of our main results.

Definition 1 (see [9]) The complex network system is exponentially synchronized when positive numbers $\xi, \alpha$ are present, so every error vector $e(t, \phi)$ of the system satisfies

$$
\|e(t, \phi)\| \leq \xi\|\phi\| e^{-\alpha\left(t-t_{0}\right)}, \quad \forall t \geq t_{0} \geq 0 .
$$

Lemma 1 (Cauchy inequality [9]) For any vector $x, y \in \mathbb{R}^{n}$ and positive definite matrix $W \in \mathbb{R}^{N \times N}$, the following matrix inequality holds:

$$
\pm 2 x^{T} y \leq x^{T} W x+y^{T} W^{-1} y .
$$

Lemma 2 (Razumikhin stability theorem [28]) Assume that $v, u, \omega: \mathbb{R}^{+} \rightarrow \mathbb{R}^{+}$are nondecreasing, and $u(v), v(v)$ are positive for $v \geq 0, v(0)=u(0)=0$, and $q>1$. If a function $V(t, e): \mathbb{R}^{+} \times \mathbb{R}^{+} \rightarrow \mathbb{R}^{+}$exists, so

(i) $u(\|e\|) \leq V(t, e) \leq v(\|e\|), t \in \mathbb{R}^{+}, e \in \mathbb{R}^{n}$,

(ii) $\dot{V}(t, e(t)) \leq-\omega(\|e\|)$, if $V(t+v, e(t+v)) \leq q V(t, e(t)), \forall v \in[-\tau, 0], t \geq 0$, then the error system is asymptotically stable.

Lemma 3 (Schur complement [29]) The matrix $\left[\begin{array}{ll}S_{11} & S_{12} \\ S_{21} & S_{22}\end{array}\right]<0$, where $S_{11}=S_{11}^{T}, S_{12}=S_{21}^{T}$ is equivalent to any one of the following conditions:

(1) $S_{22}<0, S_{11}-S_{12} S_{22}^{-1} S_{12}^{T}<0$;

(2) $S_{11}<0, S_{22}-S_{12}^{T} S_{11}^{-1} S_{12}<0$. 
Assumption 1 (see [30]) For the vector function $f(x(t), x(t-\tau(t)))$, suppose that the uniform Lipschitz condition holds and $L_{1}$ and $L_{2}$ are positive numbers such that

$$
\begin{aligned}
& \left\|f\left(x_{i}(t), x_{i}(t-\tau(t))\right)-f(s(t), s(t-\tau(t)))\right\| \\
& \quad \leq L_{1}\left\|x_{i}(t)-s(t)\right\|+L_{2}\left\|x_{i}(t-\tau(t))-s(t-\tau(t))\right\| .
\end{aligned}
$$

In particular, we have

$$
\left\|f\left(x_{i}(t)\right)-f(s(t))\right\| \leq L_{1}\left\|x_{i}(t)-s(t)\right\|,
$$

where $i=1,2, \ldots, N$.

Remark 1 In some studies on complex network synchronization problems, the effects of time delay were not considered. For example Jin-Liang Wang discussed the output synchronization and $H_{\infty}$ output synchronization of multi-weighted complex network in [31] but did not consider the network model with time delay. Moreover, many studies on complex network often require derivable or bounded time delay [20-27]. In the proposed model, the requirements for time delay $(\tau(t))$ are reduced, and the time delay does not need to be differentiated.

Remark 2 At present, there are many research results on the synchronous control of linearly coupled complex network models [31-33], but their research methods are different from ours.

\section{Main result}

\subsection{Synchronization control of a general complex dynamical network with} dynamic behavior including time-varying delays and linear time-varying coupling delay consisting of $\boldsymbol{N}$ identical nodes with linear couplings

Theorem 1 The complex network system (1) is exponentially synchronized if positive numbers $\beta, \delta, \lambda, L_{1}, L_{2}$ and a positive matrix $P$ are present and the following inequality equation holds:

$$
\begin{aligned}
& \lambda^{-1}(P+\beta I) \geq\left(c_{1} A \otimes \Gamma_{1}\right)^{T}\left(c_{1} A \otimes \Gamma_{1}\right), \\
& \lambda^{-1}(P+\beta I) \geq\left(c_{2} B \otimes \Gamma_{2}\right)^{T}\left(c_{2} B \otimes \Gamma_{2}\right), \quad \forall \delta>0, \\
& \lambda^{-1}(P+\beta I) \geq\left(L_{1}^{2}+\frac{1}{2} L_{1} L_{2}\right), \quad \lambda^{-1}(P+\beta I) \geq\left(L_{2}^{2}+\frac{1}{2} L_{1} L_{2}\right), \\
& {\left[\begin{array}{ccc}
\gamma_{1} & (P+\beta I)^{T} & \left(c_{2} B \otimes \Gamma_{2}\right)^{T}(P+\beta I) \\
(P+\beta I) & -3 I & 0 \\
\left(c_{2} B \otimes \Gamma_{2}\right)^{T}(P+\beta I) & 0 & -3 \tau I
\end{array}\right] \leq 0,}
\end{aligned}
$$

where $\gamma_{1}=\left[4 \lambda^{-1}+4 \lambda^{-1} \tau+2 K\right](P+\beta I)+\delta I$.

Moreover, the error vector $e(t, \phi)$ satisfies the condition

$$
\|e(t, \phi)\| \leq \sqrt{\frac{p+\beta}{\beta}} e^{-\frac{\delta}{2(p+\beta)} t}, \quad t \geq 0
$$

Here $p=\|P\|$. 
Proof Consider the following Lyapunov function:

$$
V=e^{T} P e+\beta e^{T} e=e^{T}(P+\beta I) e,
$$

where $P$ is a positive symmetric matrix. It is easy to see that

$$
\beta\|e\|^{2} \leq V(t, e) \leq(p+\beta)\|e\|^{2}, \quad \forall t \in \mathbb{R}^{+}, e \in \mathbb{R}^{n}
$$

For the chosen number $\lambda>0$, we have

$$
\begin{aligned}
& \lambda e^{T}\left(c_{1} A \otimes \Gamma_{1}\right)^{T}\left(c_{1} A \otimes \Gamma_{1}\right) e \leq e^{T}(P+\beta I) e, \\
& \lambda e^{T}\left(c_{2} B \otimes \Gamma_{2}\right)^{T}\left(c_{2} B \otimes \Gamma_{2}\right) e \leq e^{T}(P+\beta I) e, \\
& \lambda e^{T}\left(L_{1}^{2}+\frac{1}{2} L_{1} L_{2}\right) e \leq e^{T}(P+\beta I) e, \quad \lambda e^{T}\left(L_{2}^{2}+\frac{1}{2} L_{1} L_{2}\right) e \leq e^{T}(P+\beta I) e .
\end{aligned}
$$

We get the following inequality:

$$
\begin{aligned}
\dot{V}(t, e(t))= & 2 e^{T}(P+\beta I) \dot{e} \\
= & 2 e^{T}(P+\beta I)\left(\tilde{f}(x(t), x(t-\tau(t)))+K e(t)+c_{1} A \otimes \Gamma_{1} e(t)+c_{2} B \otimes \Gamma_{2} e(t)\right) \\
& -2 e^{T}(P+\beta I) c_{2} B \otimes \Gamma_{2} \int_{t-\tau(t)}^{t} \tilde{f}(x(v), x(v-\tau(v))) d v \\
& -2 e^{T}(P+\beta I) c_{2} B \otimes \Gamma_{2} \int_{t-\tau(t)}^{t}\left(c_{1} A \otimes \Gamma_{1} e(v)+c_{2} B \otimes \Gamma_{2} e(v-\tau(v))\right) d v .
\end{aligned}
$$

Therefore, the following estimates hold by applying Lemma 2 with $W=I$, and by taking $q \rightarrow 1^{+}$:

$$
\begin{aligned}
& 2 e^{T}(P+\beta I) \tilde{f}(x(t), x(t-\tau(t))) \\
& \quad \leq e^{T}(P+\beta I)^{2} e+\|\tilde{f}(x(t), x(t-\tau(t)))\|^{2} \\
& \quad \leq e^{T}(P+\beta I)^{2} e+\left(L_{1}\|e\|+L_{2}\|e(t-\tau(t))\|\right)^{2} \\
& \quad \leq e^{T}(P+\beta I)^{2} e+\left(L_{1}^{2}+\frac{1}{2} L_{1} L_{2}\right) e^{T} e+\left(L_{2}^{2}+\frac{1}{2} L_{1} L_{2}\right) e^{T}(t-\tau(t)) e(t-\tau(t)), \\
& 2 e^{T}(P+\beta I) c_{2} B \otimes \Gamma_{2} e(t) \\
& \left.\quad \leq e^{T}(P+\beta I)(P+\beta I) e+\lambda^{-1} V(t)\right), \\
& 2 e^{T}(P+\beta I) c_{1} A \otimes \Gamma_{1} e(t) \\
& \quad \leq e^{T}(P+\beta I)(P+\beta I) e+\lambda^{-1} V(t), \\
& -2 e^{T}(P+\beta I) c_{2} B \otimes \Gamma_{2} \int_{t-\tau(t)}^{t} \tilde{f}(x(v), x(v-\tau(v))) d v \\
& \quad \leq \tau e^{T}(P+\beta I) c_{2} B \otimes \Gamma_{2}\left(c_{2} B \otimes \Gamma_{2}\right)^{T}(P+\beta I) e+\int_{t-\tau(t)}^{t}\|\tilde{f}(x, x(v-\tau(v)))\|^{2} d v \\
& \leq \tau e^{T}(P+\beta I) c_{2} B \otimes \Gamma_{2}\left(c_{2} B \otimes \Gamma_{2}\right)^{T}(P+\beta I) e
\end{aligned}
$$




$$
\begin{aligned}
& +\int_{t-\tau(t)}^{t}\left(L_{1}^{2}+\frac{1}{2} L_{1} L_{2}\right) e^{T} e+\left(L_{2}^{2}+\frac{1}{2} L_{1} L_{2}\right) e^{T}(t-\tau(t)) e(t-\tau(t)) d v \\
\leq & \tau e^{T}(P+\beta I) c_{2} B \otimes \Gamma_{2}\left(c_{2} B \otimes \Gamma_{2}\right)^{T}(P+\beta I) e+2 \lambda^{-1} \tau V(t) \\
2 e^{T}(P+\beta I) K e=K V(t) & \\
-2 & e^{T}(P+\beta I) c_{2} B \otimes \Gamma_{2} \int_{t-\tau(t)}^{t} c_{1} A \otimes \Gamma_{1} e(v) d v \\
= & -\int_{t-\tau(t)}^{t} 2 e^{T}(P+\beta I) c_{2} B \otimes \Gamma_{2} c_{1} A \otimes \Gamma_{1} e(v) d v \\
\leq & \tau e^{T}(P+\beta I) c_{2} B \otimes \Gamma_{2}\left(c_{2} B \otimes \Gamma_{2}\right)^{T}(P+\beta I) e \\
& +\int_{t-\tau(t)}^{t} e^{T}(v)\left(c_{1} A \otimes \Gamma_{1}\right)^{T} c_{1} A \otimes \Gamma_{1} e(v) d v \\
\leq & \tau e^{T}(P+\beta I) c_{2} B \otimes \Gamma_{2}\left(c_{2} B \otimes \Gamma_{2}\right)^{T}(P+\beta I) e+\lambda^{-1} \int_{t-\tau(t)}^{t} e^{T}(v)(P+\beta I) e(v) d v \\
\leq & \tau e^{T}(P+\beta I) c_{2} B \otimes \Gamma_{2}\left(c_{2} B \otimes \Gamma_{2}\right)^{T}(P+\beta I) e+\lambda^{-1} \int_{-\tau(t)}^{0} e^{T}(t+v)(P+\beta I) e(t+v) d v \\
\leq & \tau e^{T}(P+\beta I) c_{2} B \otimes \Gamma_{2}\left(c_{2} B \otimes \Gamma_{2}\right)^{T}(P+\beta I) e+\lambda^{-1} \tau V(t)
\end{aligned}
$$

In a similar way

$$
\begin{aligned}
& -2 e^{T}(P+\beta I) c_{2} B \otimes \Gamma_{2} \int_{t-\tau(t)}^{t} c_{2} B \otimes \Gamma_{2} e(v-\tau(v)) d v \\
& \leq \tau e^{T}(P+\beta I) c_{2} B \otimes \Gamma_{2}\left(c_{2} B \otimes \Gamma_{2}\right)^{T}(P+\beta I) e+\lambda^{-1} \tau V(t) .
\end{aligned}
$$

According to the above proof, we get

$$
\begin{aligned}
\dot{V} \leq & e^{T}\left[4(P+\beta I)^{2}+\left(4 \lambda^{-1}+4 \lambda^{-1} \tau\right)(P+\beta I)\right] e+2 e(P+\beta I) K e \\
& +e^{T}\left[3 \tau(P+\beta I) c_{2} B \otimes \Gamma_{2}\left(c_{2} B \otimes \Gamma_{2}\right)^{T}(P+\beta I)\right] e .
\end{aligned}
$$

Assume $\dot{V} \leq-\delta e^{T} e$, where $\forall \delta>0$, it means $\dot{V}<0$, therefore

$$
\begin{aligned}
& 3(P+\beta I)^{2}+\left(4 \lambda^{-1}+4 \lambda^{-1} \tau\right)(P+\beta I)+2 e(P+\beta I) K e \\
& \quad+3 \tau(P+\beta I) c_{2} B \otimes \Gamma_{2}\left(c_{2} B \otimes \Gamma_{2}\right)^{T}(P+\beta I)+\delta I \leq 0 .
\end{aligned}
$$

According to Lemma 3, it is equivalent to the following matrix inequalities:

$$
\left[\begin{array}{ccc}
\gamma_{1} & (P+\beta I)^{T} & \left(c_{2} B \otimes \Gamma_{2}\right)^{T}(P+\beta I) \\
(P+\beta I) & -3 I & 0 \\
\left(c_{2} B \otimes \Gamma_{2}\right)^{T}(P+\beta I) & 0 & -3 \tau I
\end{array}\right] \leq 0 .
$$

Here $\gamma_{1}=\left[4 \lambda^{-1}+4 \lambda^{-1} \tau+2 K\right](P+\beta I)+\delta I$ and

$$
\dot{V} \leq-\delta e^{T} e=-\delta\|e\|^{2} .
$$


This, by the Razumikhin stability theorem and Lemma 2, implies the asymptotic stability of the error system (6). To find the exponential factor of the solution, integrating both sides of the inequality, due to (16), $\dot{V}(t, e(t)) \leq 0$ and using the condition (8), we have

$$
\beta\|e\|^{2} \leq V(t, e) \leq(p+\beta)\|e\|^{2}
$$

such that

$$
\beta\|e(t, \phi)\|^{2} \leq V(t, e(t)) \leq V(0, e(0))^{-\frac{\delta}{2(p+\beta)}}, \quad t \geq 0 .
$$

Hence

$$
\|e(t, \phi)\| \leq \sqrt{\frac{p+\beta}{\beta}} e^{-\frac{\delta}{2(p+\beta)} t}, \quad t \geq 0 .
$$

Here $p=\|P\|$, this completes the proof of Theorem 1 .

Remark 3 Although many studies on the synchronous control of complex network systems with time-varying delay have generated many and variable conclusions, the studies, especially those regarding the synchronization stability of systems, all used the same two requirements on time-varying delay. First, a time-varying delay should have boundaries; that is, $0 \leq \tau(t) \leq \tau, \forall t>0$. Second, the time-varying delay should be derivable and smaller than 1 ; that is, $0 \leq \dot{\tau}(t) \leq 1, \forall t>0$. For example [22,25], the time-varying delay may not be derivable. In fact, the time-varying delay is not derivable when it changes quickly in a time period. In this study, only the first requirement of time-varying delay is needed. The second requirement can be solved by the Newton-Leibniz formula. The stability conditions of the exponential synchronization of the system are obtained through the Razumikhin theorem. Therefore, this theorem relieves the requirements to the system in contrast to the theorems used in other studies [20-27].

Remark 4 In [34, 35], the synchronization (or stability) of similar models, which all require a derivable time-varying delay, is investigated. Similarly, this requirement is unnecessary in Corollary 2.

As a special case, for the system (17), we consider the following Lyapunov function:

$$
V=e^{T} P e
$$

According to the proof of Theorem 1, it is easy to get the following conclusion. We have the following corollary.

Corollary 1 The complex network system (1) is exponentially synchronized if positive numbers $\beta, \delta, \lambda, L_{1}, L_{2}$, and a positive matrix $P$ exist such that the following inequality equation holds:

$$
\begin{aligned}
& \lambda^{-1} P \geq\left(c_{1} A \otimes \Gamma_{1}\right)^{T}\left(c_{1} A \otimes \Gamma_{1}\right), \quad \lambda^{-1} P \geq\left(c_{2} B \otimes \Gamma_{2}\right)^{T}\left(c_{2} B \otimes \Gamma_{2}\right), \quad \forall \delta>0, \\
& \lambda^{-1} P \geq\left(L_{1}^{2}+\frac{1}{2} L_{1} L_{2}\right), \quad \lambda^{-1} P \geq\left(L_{2}^{2}+\frac{1}{2} L_{1} L_{2}\right),
\end{aligned}
$$




$$
\left[\begin{array}{ccc}
\gamma_{2} & P^{T} & \left(c_{2} B \otimes \Gamma_{2}\right)^{T} P \\
P & -4 I & 0 \\
\left(c_{2} B \otimes \Gamma_{2}\right)^{T} P & 0 & -3 \tau I
\end{array}\right] \leq 0,
$$

where $\gamma_{2}=\left[4 \lambda^{-1}+4 \lambda^{-1} \tau+2 K\right] P+\delta I$.

Moreover, the error vector $e(t, \phi)$ satisfies the condition

$$
\|e(t, \phi)\| \leq \sqrt{\frac{p}{\beta}} e^{-\frac{\delta}{2 p} t}, \quad t \geq 0 .
$$

Here $p=\|P\|$.

Remark 5 In Corollary $1, \beta e^{T} e$ is removed from the Lyapunov function by proving Theorem 1, which simplifies the judgment on synchronization stability and requires fewer parameters. However, this approach increases the difficulty of selecting the parameter $\lambda$.

\subsection{The synchronization control of complex dynamical network with time-varying coupling delay consisting of $N$ identical nodes with linear couplings}

In this section, we consider a general complex dynamical network consisting of dynamical nodes with linear couplings, which is described by

$$
\begin{aligned}
& \dot{x}_{i}=f\left(x_{i}(t)\right)+c_{1} \sum_{j=1}^{N} a_{i j} \Gamma_{1} x_{j}(t)+c_{2} \sum_{j=1}^{N} b_{i j} \Gamma_{2} x_{j}(t-\tau(t)), \\
& x_{i}(t)=\phi(t), \quad t \in[-\tau, 0], i=1,2, \ldots, N,
\end{aligned}
$$

$x_{i}=\left(x_{i 1}(t), x_{i 2}(t), \ldots, x_{i n}(t)\right)^{T} \in \mathbb{R}^{n}$ is the state vector of the $i$ th leader, $f: \mathbb{R}^{n} \times \mathbb{R}^{n} \rightarrow$ $\mathbb{R}^{n}$ is a continuously differential nonlinear vector function, $c_{1}>0$ and $c_{2}>0$ are the non-delay and time-varying delay coupling strengths, $\Gamma_{1}=\operatorname{diag}\left(\gamma_{1}^{1}, \gamma_{1}^{2}, \ldots, \gamma_{1}^{n}\right)$ and $\Gamma_{2}=$ $\operatorname{diag}\left(\gamma_{2}^{1}, \gamma_{2}^{2}, \ldots, \gamma_{2}^{n}\right)$ are positive definite diagonal matrices, which represent the inner connection matrices between each pair of nodes; $A=\left(a_{i j}\right) \in \mathbb{R}^{N \times N}, B=\left(b_{i j}\right) \in \mathbb{R}^{N \times N}$ are the non-delay and time-varying delay weight matrices. The entries are defined as follows: if is a link is present from the node $i$ to the node $j(i \neq j)$, then $a_{i j} \neq 0, b_{i j} \neq 0$; otherwise $a_{i j}=0$, $b_{i j}=0$, and the diagonal elements of matrices $A$ and $B$ are defined as $a_{i i}=-\sum_{j=1, j \neq i}^{N} a_{i j}$, $b_{i i}=-\sum_{j=1, j \neq i}^{N} b_{i j}$. Given that the networks in this paper are direct, $a_{i j} \neq a_{j i}$ and $b_{i j} \neq b_{j i}$ $(i, j=1,2, \ldots, N)$. The coupling time-varying delay $\tau(t)$ is a bound function and a positive constant $\tau$ satisfying $0 \leq \tau(t) \leq \tau, \forall t>0, u_{i} \in \mathbb{R}^{N \times N}$ is the control input of node $i$. In this paper, we aim to design a suitable controller,

$$
u_{i}(t)=k_{i}\left(x_{i}(t)-s(t)\right)
$$

where $k_{i}$ is the gain of the $i$ th node.

We use the Newton-Leibniz formula

$$
x(t-\tau(t))=x(t)-\int_{t-\tau(t)}^{t} \dot{x}(s) d s .
$$


We convert Eq. (21) to

$$
\begin{aligned}
& \dot{x}_{i}=f(x(t))+c_{1} A \otimes \Gamma_{1} x+c_{2} B \otimes \Gamma_{2}\left[x(t)-\int_{t-\tau(t)}^{t} \dot{x}(s) d s\right], \\
& x(t)=\psi(t), \quad t \in[-2 \tau, 0] .
\end{aligned}
$$

The set $s=\left\{x_{1}^{T}(t), x_{2}^{T}(t), \ldots, x_{n}^{T}(t) \in \mathbb{R}^{n} \mid x_{i}(t)=s(t), i \in \mathbb{N}\right\}$ is defined as a synchronization manifold, where $s(t) \in \mathbb{R}^{n}$ satisfies

$$
\dot{s}(t)=f(s(t))
$$

Let $\tilde{f}(x)=f(x)-f(s(t)), K=\operatorname{diag}\left(k_{1}, k_{2}, \ldots, k_{N}\right)$.

Define the synchronization error vector as

$$
e_{i}(t)=\left(x_{i}(t)-s(t), \quad e_{i}(t-\tau(t))=x_{i}(t-\tau(t))-s(t-\tau(t)) \quad(i=1, \ldots, N)\right.
$$

Through (22) and (23), in order to achieve the synchronization of system (20), we add controller (21) to the error system, we can get the following equation:

$$
\begin{aligned}
\dot{e}(t)= & \tilde{f}(x)+c_{1} A \otimes \Gamma_{1} e+c_{2} B \otimes \Gamma_{2}\left[e(t)-\int_{t-\tau(t)}^{t} \dot{e}(v) d v\right]+K e(t) \\
= & \tilde{f}(x)+\left(c_{1} A \otimes \Gamma_{1}+c_{2} B \otimes \Gamma_{2}\right) e(t) \\
& -c_{2} B \otimes \Gamma_{2} \int_{t-\tau(t)}^{t}\left[\tilde{f}(v)+c_{1} A \otimes \Gamma_{1}+c_{2} B \otimes \Gamma_{2} e(v-\tau(v))\right] d v+K e(t),
\end{aligned}
$$

where $e_{i}(t)=\left(e_{i 1}(t), e_{i 2}(t), \ldots, e_{i n}(t)\right)^{T} \in \mathbb{R}^{n}, e(t)=\left(e_{1}(t), e_{2}(t), \ldots, e_{n}(t)\right)^{T} \in \mathbb{R}^{n}$.

Therefore, if the error system (24) is exponentially stable, the complex network system (20) is exponentially synchronized.

Theorem 2 The complex network system (18) is exponentially synchronized if positive numbers $\beta, \delta, \lambda, L_{1}$, and a positive matrix $P$ exist such that the following inequality equation holds:

$$
\begin{aligned}
& \lambda^{-1}(P+\beta I) \geq\left(c_{1} A \otimes \Gamma_{1}\right)\left(c_{1} A \otimes \Gamma_{1}\right)^{T}, \\
& \lambda^{-1}(P+\beta I) \geq\left(c_{2} B \otimes \Gamma_{2}\right)\left(c_{2} B \otimes \Gamma_{2}\right)^{T}, \quad \forall \delta>0, \\
& {\left[\begin{array}{ccc}
\gamma_{3} & (P+\beta I)^{T} & \left(c_{2} B \otimes \Gamma_{2}\right)^{T}(P+\beta I) \\
(P+\beta I) & -3 I & 0 \\
\left(c_{2} B \otimes \Gamma_{2}\right)^{T}(P+\beta I) & 0 & -3 \tau I
\end{array}\right] \leq 0,}
\end{aligned}
$$

where $\gamma_{3}=L_{1}^{2} I+(1+4 \tau) \lambda^{-1}(P+\beta I)+\delta I+2 K(P+\beta I)$.

Moreover, the error vector $e(t, \phi)$ satisfies the condition

$$
\|e(t, \phi)\| \leq \sqrt{\frac{p+\beta}{\beta}} e^{-\frac{\delta}{2(p+\beta)} t}, \quad t \geq 0 .
$$

Here $p=\|P\|$. 
Proof We consider the following Lyapunov function:

$$
V=e^{T} P e+\beta e^{T} e=e^{T}(P+\beta I) e .
$$

Here $P$ is a positive symmetric matrix. It is easy to see that

$$
\beta\|e\|^{2} \leq V(t, e) \leq(p+\beta)\|e\|^{2}, \quad \forall t \in R^{+}, e \in \mathbb{R}^{n} .
$$

The time derivative of $V(t, x)$ along the trajectory of the system (22) is given by

$$
\begin{aligned}
\dot{V}(t, e(t))= & 2 e^{T} P \dot{e}+2 \beta e^{T} \dot{e} \\
= & 2 e^{T}(P+\beta I) \dot{e} \\
= & 2 e^{T}(P+\beta I)\left(\tilde{f}(x(t))+K e(t)+\left(c_{1} A \otimes \Gamma_{1}+c_{2} B \otimes \Gamma_{2}\right) e(t)\right) \\
& -2 e^{T}(P+\beta I) c_{2} B \otimes \Gamma_{2} \int_{t-\tau(t)}^{t} \tilde{f}(x(v)) d v \\
& -2 e^{T}(P+\beta I) \\
& \times c_{2} B \otimes \Gamma_{2} \int_{t-\tau(t)}^{t}\left(c_{1} A \otimes \Gamma_{1} e(v)+c_{2} B \otimes \Gamma_{2} e(v-\tau(v))\right) d v .
\end{aligned}
$$

For the chosen number $\lambda>0$, we have

$$
\begin{aligned}
& \lambda e^{T}\left(c_{1} A \otimes \Gamma_{1}\right)^{T}\left(c_{1} A \otimes \Gamma_{1}\right) e \leq e^{T}(P+\beta I) e, \\
& \lambda e^{T}\left(c_{2} B \otimes \Gamma_{2}\right)^{T}\left(c_{2} B \otimes \Gamma_{2}\right) e \leq e^{T}(P+\beta I) e .
\end{aligned}
$$

Therefore, the following estimates hold by applying Lemma 1 with $W=I$ :

$$
\begin{aligned}
2 e^{T}(P+\beta I)(f(x)-f(s(t))) & \leq e^{T}(P+\beta I)(P+\beta I) e+\|f(x)-f(s(t))\|^{2} \\
& \leq e^{T}(P+\beta I)(P+\beta I) e+L_{1}^{2} e^{T} e .
\end{aligned}
$$

In the light of the Razumikhin theorem, we assume that for any real number $q>1$

$$
V(t+s, e(s+t)) \leq q V(t, e(t)), \quad \forall s \in[-2 \tau, 0], \forall t \geq 0,
$$

and taking $q \rightarrow 1^{+}$leads to

$$
\begin{aligned}
& -2 e^{T}(P+\beta I) c_{2} B \otimes \Gamma_{2} \int_{t-\tau(t)}^{t} \tilde{f}(x(v) d v \\
& \leq \tau e^{T}(P+\beta I) c_{2} B \otimes \Gamma_{2}\left(c_{2} B \otimes \Gamma_{2}\right)^{T}(P+\beta I) e+\int_{t-\tau(t)}^{t}\|\tilde{f}(x(v))\|^{2} d v \\
& \leq \tau e^{T}(P+\beta I) c_{2} B \otimes \Gamma_{2}\left(c_{2} B \otimes \Gamma_{2}\right)^{T}(P+\beta I) e+\int_{t-\tau(t)}^{t} L_{1}^{2} e^{T} e d v \\
& \leq \tau e^{T}(P+\beta I) c_{2} B \otimes \Gamma_{2}\left(c_{2} B \otimes \Gamma_{2}\right)^{T}(P+\beta I) e+\lambda^{-1} \tau V(t),
\end{aligned}
$$




$$
\begin{aligned}
- & 2 e^{T}(P+\beta I) c_{2} B \otimes \Gamma_{2} \int_{t-\tau(t)}^{t} c_{1} A \otimes \Gamma_{1} e(v) d v \\
= & -\int_{t-\tau(t)}^{t} 2 e^{T}(P+\beta I) c_{2} B \otimes \Gamma_{2} c_{1} A \otimes \Gamma_{1} e(v) d v \\
\leq & \tau e^{T}(P+\beta I) c_{2} B \otimes \Gamma_{2}\left(c_{2} B \otimes \Gamma_{2}\right)^{T}(P+\beta I) e \\
& +\int_{t-\tau(t)}^{t} e^{T}(v)\left(c_{1} A \otimes \Gamma_{1}\right)^{T} c_{1} A \otimes \Gamma_{1} e(v) d v \\
\leq & \tau e^{T}(P+\beta I) c_{2} B \otimes \Gamma_{2}\left(c_{2} B \otimes \Gamma_{2}\right)^{T}(P+\beta I) e+\lambda^{-1} \int_{t-\tau(t)}^{t} e^{T}(v)(P+\beta I) e(v) d v \\
\leq & \tau e^{T}(P+\beta I) c_{2} B \otimes \Gamma_{2}\left(c_{2} B \otimes \Gamma_{2}\right)^{T}(P+\beta I) e+\lambda^{-1} \int_{-\tau(t)}^{0} e^{T}(t+v)(P+\beta I) e(t+v) d v \\
\leq & \tau e^{T}(P+\beta I) c_{2} B \otimes \Gamma_{2}\left(c_{2} B \otimes \Gamma_{2}\right)^{T}(P+\beta I) e+\lambda^{-1} \tau V(t) .
\end{aligned}
$$

In a similar way

$$
\begin{aligned}
& -2 e^{T}(P+\beta I) c_{2} B \otimes \Gamma_{2} \int_{t-\tau(t)}^{t} c_{2} B \otimes \Gamma_{2} e(v-\tau(v)) d v \\
& \quad \leq \tau e^{T}(P+\beta I) c_{2} B \otimes \Gamma_{2}\left(c_{2} B \otimes \Gamma_{2}\right)^{T}(P+\beta I) e+\lambda^{-1} \tau V(t), \\
& 2 e^{T}(P+\beta I)\left(c_{1} A \otimes \Gamma_{1}+c_{2} B \otimes \Gamma_{2}\right) e \\
& \quad \leq 2 e^{T}(P+\beta I)(P+\beta I) e+2 \lambda^{-1} e^{T}(P+\beta I) e .
\end{aligned}
$$

Therefore

$$
\begin{aligned}
\dot{V} \leq & e^{T}\left[L_{1}^{2} I+3 \tau(P+\beta I) c_{2} B \otimes \Gamma_{2}\left(c_{2} B \otimes \Gamma_{2}\right)^{T}(P+\beta I)\right] e+2 K e^{T}(P+\beta I) e \\
& +e^{T}\left[\lambda^{-1}(1+4 \tau)(P+\beta I)\right] e+e^{T}[3(P+\beta I)(P+\beta I)] e .
\end{aligned}
$$

Assume $\dot{V} \leq-\delta e^{T} e$, where $\forall \delta>0$, which means $\dot{V}<0$, therefore

$$
\begin{aligned}
& 3(P+\beta I)(P+\beta I)+L_{1}^{2} I+3 \tau(P+\beta I) c_{2} B \otimes \Gamma_{2}\left(c_{2} B \otimes \Gamma_{2}\right)^{T}(P+\beta I) \\
& +\lambda^{-1}(1+4 \tau)(P+\beta I)+2 K(P+\beta I)+\delta I \leq 0 .
\end{aligned}
$$

According to Lemma 3, we could have transformed (36) into (37), it is equivalent to the following matrix inequalities:

$$
\left[\begin{array}{ccc}
\gamma_{3} & (P+\beta I)^{T} & \left(c_{2} B \otimes \Gamma_{2}\right)^{T}(P+\beta I) \\
(P+\beta I) & -3 I & 0 \\
\left(c_{2} B \otimes \Gamma_{2}\right)^{T}(P+\beta I) & 0 & -3 \tau I
\end{array}\right] \leq 0,
$$

where $\gamma_{3}=L_{1}^{2} I+\lambda^{-1}(1+4 \tau)(P+\beta I)+\delta I+2 K(P+\beta I)$.

Also

$$
\dot{V} \leq-\delta e^{T} e=-\delta\|e\|^{2} .
$$


This, by the Razumikhin stability theorem and Lemma 2, implies the asymptotic stability of the error system (24). To find the exponential factor of the solution, integrating both sides of the inequality, due to (39), $\dot{V}(t, e(t)) \leq 0$ and using the condition (26), we have

$$
\beta\|e\|^{2} \leq V(t, e) \leq V(0, e(0)) e^{-\frac{\delta}{2(p+\beta)} t}
$$

and hence

$$
\|e(t, \phi)\| \leq \sqrt{\frac{p+\beta}{\beta}} e^{-\frac{\delta}{2(p+\beta)} t}, \quad t \geq 0 .
$$

Here $p=\|P\|$, this completes the proof of Theorem 2 .

Remark 6 Many researchers who studied the synchronous control of complex network with time-varying delay hypothesized that dynamic behaviors include nonlinear function. Some of them did not consider time delay in nonlinear dynamic behaviors [36] and some did [37]. Notably, the time-varying delay of systems (time delay in dynamic behavior and node coupling) was considered derivable in previous studies. This condition is eliminated in our Theorem 2, which relieves the requirements on time delay and thereby reduces the conservation of conclusions.

We turn to system (18). We consider the following Lyapunov function:

$$
V=e^{T} P e .
$$

According to Theorem 2, it is easy to get the following conclusion. We have the following corollary.

Corollary 2 The complex network system (18) is exponentially synchronized when positive numbers $\delta, \lambda, L_{1}$ and a positive matrix $P$ are present and the following inequality equation holds:

$$
\begin{aligned}
& \lambda^{-1} P \geq\left(c_{1} A \otimes \Gamma_{1}\right)^{T}\left(c_{1} A \otimes \Gamma_{1}\right), \quad \lambda^{-1} P \geq\left(c_{2} B \otimes \Gamma_{2}\right)^{T}\left(c_{2} B \otimes \Gamma_{2}\right), \quad \forall \delta>0, \\
& {\left[\begin{array}{ccc}
\gamma_{4} & P^{T} & \left(c_{2} B \otimes \Gamma_{2}\right)^{T} P \\
P & -3 I & 0 \\
\left(c_{2} B \otimes \Gamma_{2}\right)^{T} P & 0 & -3 \tau I
\end{array}\right] \leq 0,}
\end{aligned}
$$

where $\gamma_{4}=L_{1}^{2} I+\lambda^{-1}(1+4 \tau) P+\delta I+2 K P$.

Moreover, the error vector $e(t, \phi)$ satisfies the condition

$$
\|e(t, \phi)\| \leq \sqrt{\frac{p}{\beta}} e(t-\tau(t))^{-\frac{\delta}{2 p} t}, \quad t \geq 0 .
$$

Here $p=\|P\|$. 


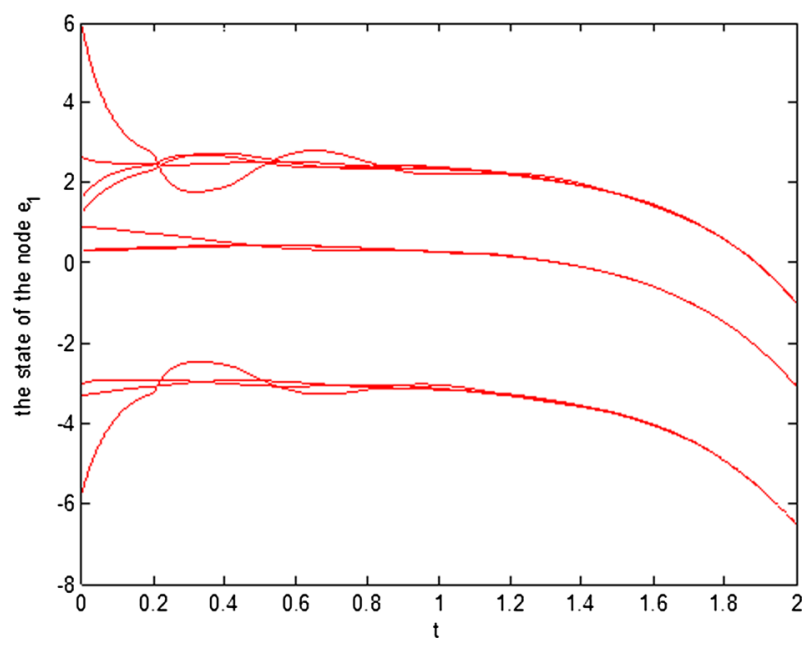

Figure 1 The state of the node $e_{1}$ without controller

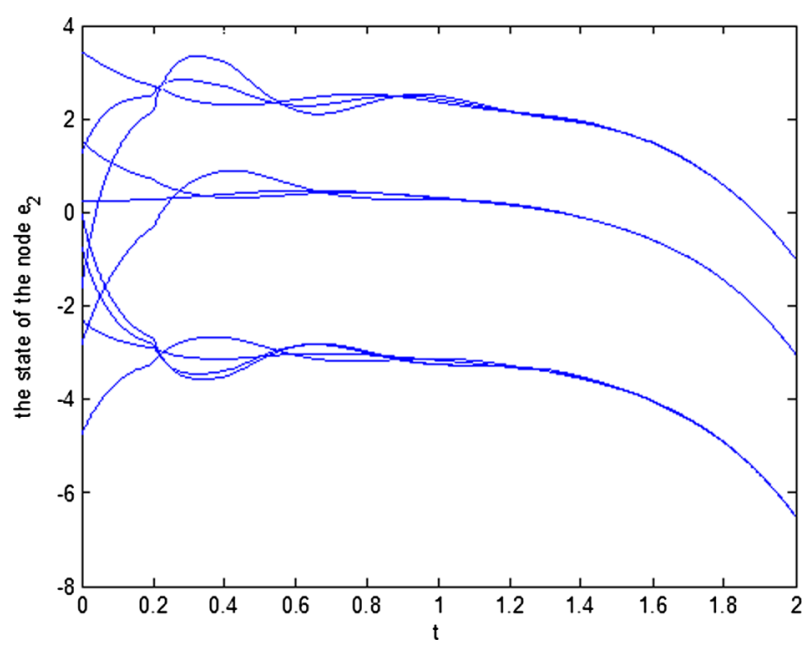

Figure 2 The state of the node $e_{2}$ without controller

\subsection{Numerical simulation}

In this section, the exponential synchronization conditions obtained in this paper are illustrated with an example.

Consider a general complex dynamical network consisting of dynamical nodes with linear couplings system in Theorem 2 . We have

$$
\dot{x}(t)=f(x(t))+c_{1} A \otimes \Gamma_{1} x(t)+c_{2} B \otimes \Gamma_{2} x(t-\tau(t))+u(t)
$$

and consider a complex network system with 10 nodes, each of which is a threedimensional linear system. Here

$$
\begin{aligned}
& c_{1}=0.4 ; \quad c_{2}=0.4 ; \quad \beta=1 ; \quad \delta=1 ; \\
& \lambda=0.001 ; \quad \tau=0.002 ; \quad L_{1}=0.1 ;
\end{aligned}
$$




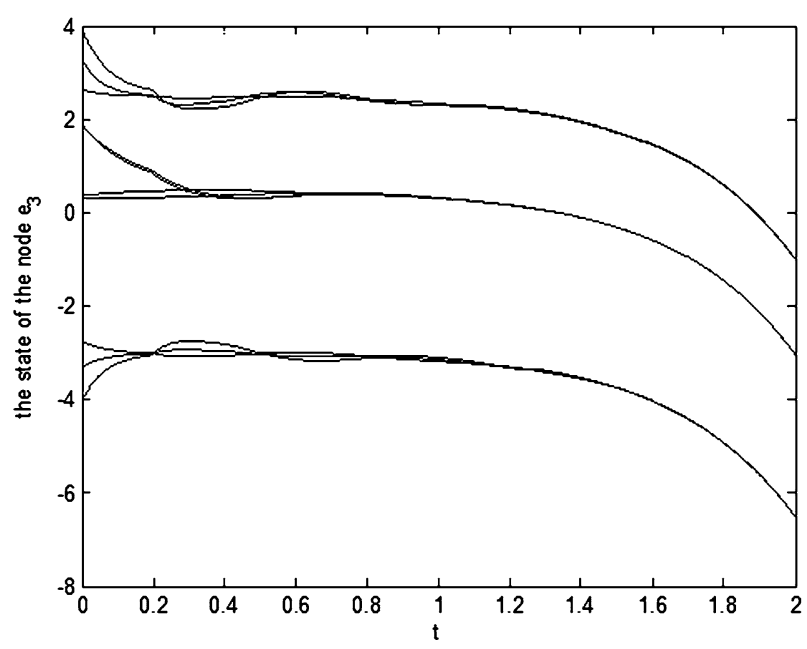

Figure 3 The state of the node $e_{3}$ without controller

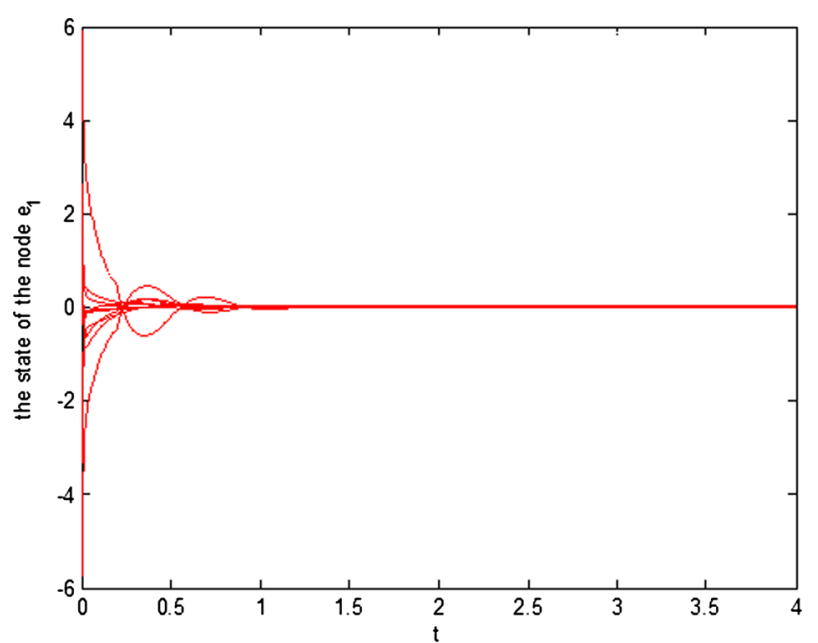

Figure 4 The state of the node $e_{1}$ with controller

$$
A=\left[\begin{array}{cccccccccc}
-7 & 1 & 1 & 0 & 1 & 1 & 1 & 0 & 1 & 1 \\
1 & -8 & 1 & 1 & 1 & 1 & 0 & 1 & 1 & 1 \\
1 & 1 & -7 & 1 & 0 & 1 & 1 & 0 & 1 & 1 \\
0 & 1 & 1 & -8 & 1 & 1 & 1 & 1 & 1 & 1 \\
1 & 1 & 0 & 1 & -7 & 1 & 0 & 1 & 1 & 1 \\
1 & 1 & 1 & 1 & 1 & -7 & 0 & 1 & 1 & 0 \\
1 & 1 & 1 & 1 & 0 & 0 & -7 & 1 & 1 & 1 \\
0 & 0 & 0 & 1 & 1 & 1 & 1 & -5 & 1 & 0 \\
1 & 1 & 1 & 1 & 1 & 1 & 1 & 1 & -9 & 1 \\
1 & 1 & 1 & 1 & 1 & 0 & 1 & 1 & 0 & -7
\end{array}\right],
$$




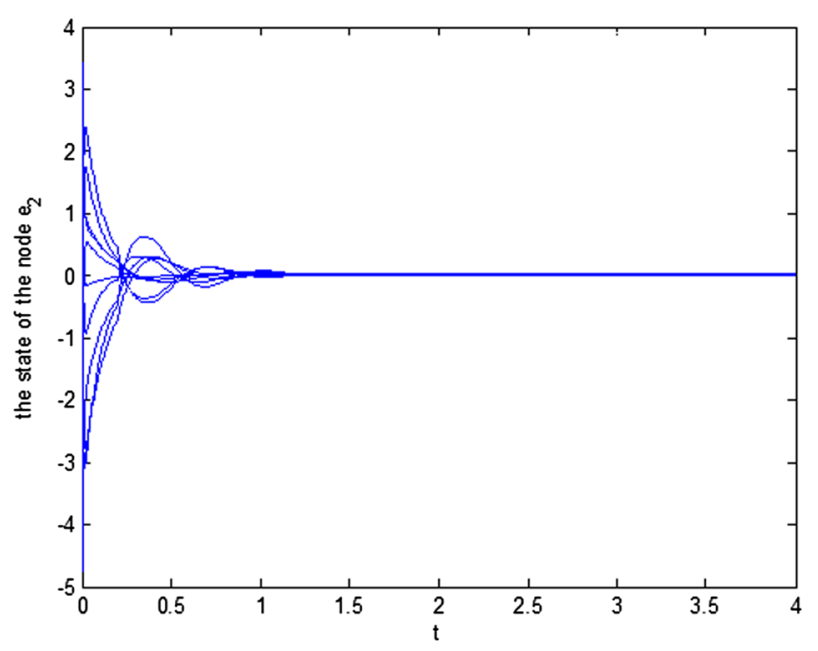

Figure $\mathbf{5}$ The state of the node $e_{2}$ with controller

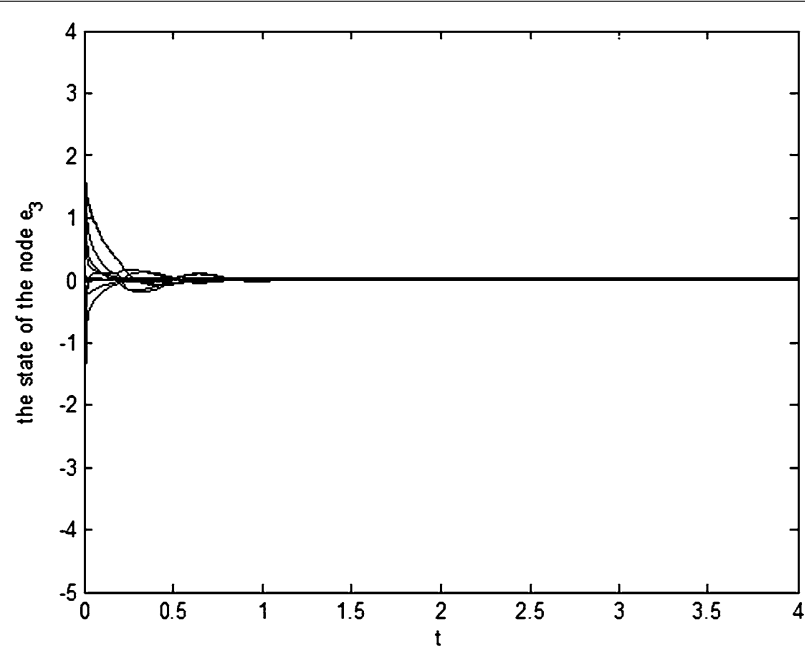

Figure 6 The state of the node $e_{3}$ with controller

$$
B=\left[\begin{array}{cccccccccc}
-7 & 1 & 1 & 0 & 1 & 1 & 1 & 0 & 1 & 1 \\
1 & -8 & 1 & 1 & 1 & 1 & 0 & 1 & 1 & 1 \\
1 & 1 & -7 & 1 & 0 & 1 & 1 & 0 & 1 & 1 \\
0 & 1 & 1 & -8 & 1 & 1 & 1 & 1 & 1 & 1 \\
1 & 1 & 0 & 1 & -7 & 1 & 0 & 1 & 1 & 1 \\
1 & 1 & 1 & 1 & 1 & -7 & 0 & 1 & 1 & 0 \\
1 & 1 & 1 & 1 & 0 & 0 & -7 & 1 & 1 & 1 \\
0 & 0 & 0 & 1 & 1 & 1 & 1 & -5 & 1 & 0 \\
1 & 1 & 1 & 1 & 1 & 1 & 1 & 1 & -9 & 1 \\
1 & 1 & 1 & 1 & 1 & 0 & 1 & 1 & 0 & -7
\end{array}\right],
$$

$\Gamma_{1}=\operatorname{diag}\{2,2,1\} ; \quad \Gamma_{2}=\operatorname{diag}\{2,2,1\}$. 
According to the LMI toolbox in Matlab, the parameters $K$ and $P$ can be obtained, and the initial conditions of the system are given, $t_{0}=0$, and the initial state $x(0)=$ $[2.62342,-5.74317, \ldots, 2.62342,-2.74317,1.89187]^{T}$ is given at random. Figures 1,2 , and 3 are for the node 1, 2, 3 status curves without controller and Figs. 4, 5, and 6 are for the node 1, 2, 3 status curves with controller.

As can be seen from the figure, after a period of time, the system state is synchronous, and the feasibility and effectiveness of the control method are demonstrated.

\section{Conclusions and discussions}

This work focuses on the problem of exponential synchronization in complex network systems with time-varying delay. In the light of the Razumikhin stability theorem combined with the Newton-Leibniz formula, we deduced an exponential synchronization condition, without considering the differentiability of the time-delay function. Finally, a numerical simulation is given to show its effectiveness. The following will be considered in our future work: how to achieve synchronization through a network that is less strict and sufficient, and how to extend existing methods to the problem of finite-time synchronization or fixed-time synchronization. This is the goal we will consider and study in the future.

Acknowledgements

The authors would like to thank X. Zou for her great help in many aspects.

Funding

This work was jointly supported by the National Natural Science Foundation of China (Grant No. 11372107), the Natural Science Foundation of Hunan Province (Grant No. 2017JJ5011) and the Natural Science Foundation of Hunan Province (Grant No. 2017JJ4004).

Availability of data and materials

The datasets used or analyzed during the current study are available from the corresponding author on request.

Competing interests

The authors declare that they have no competing interests.

Authors' contributions

All authors contributed equally to the writing of this paper. Furthermore, all authors also read carefully and approved the final manuscript.

\section{Publisher's Note}

Springer Nature remains neutral with regard to jurisdictional claims in published maps and institutional affiliations.

Received: 30 September 2018 Accepted: 3 January 2019 Published online: 14 January 2019

\section{References}

1. Steinmetz, N.A., Moore, T.: Eye movement preparation modulates neuronal responses in area V4 when dissociated from attentional demands. Neuron 83(2), 496-506 (2014)

2. Steinmetz, P.N., Roy, A., Fitzgerald, P.J., et al.: Attention modulates synchronized neuronal firing in primate somatosensory cortex. Nature 404(6774), 187 (2000)

3. Millérioux, G., Daafouz, J.: Chaos synchronization: from the genesis to polytopic observers. IFAC Proc. Vol. 39, 335-340 (2006)

4. Kuhnert, L., Agladze, K.I., Krinsky, V.I.: Image processing using light-sensitive chemical waves. Nature 337(6204), 244-247 (1989)

5. Jiang, S., Lu, X., Xie, C., Cai, S.: Adaptive finite-time control for overlapping cluster synchronization in coupled complex networks. Neurocomputing 266, 188-195 (2017)

6. Yang, M., Wang, Y.-W., Xiao, J.-W., Huang, Y.: Robust synchronization of singular complex switched networks with parametric uncertainties and unknown coupling topologies via impulsive control. Commun. Nonlinear Sci. Numer. Simul. 17(11), 4404-4416 (2012)

7. Fan, Y., Liu, H., Zhu, Y., Mei, J.: Fast synchronization of complex dynamical networks with time-varying delay via periodically intermittent control. Neurocomputing 205, 182-194 (2016)

8. Xu, M., Wang, J.-L., Huang, Y.-L., Wei, P.-C., Wang, S.-X.: Pinning synchronization of complex dynamical networks with and without time-varying delay. Neurocomputing 266, 263-273 (2017)

9. Phat, V.N., Le Hien, V.: An application of Razumikhin theorem to exponential stability for linear non-autonomous systems with time-varying delay. Appl. Math. Lett. 22(9), 1412-1417 (2009) 
10. Zhang, X., Li, D., Zhang, X.: Adaptive fuzzy impulsive synchronization of chaotic systems with random parameters. Chaos Solitons Fractals 104, 77-83 (2017)

11. Ahmed, M.A.A., Liu, Y., Zhang, W., Alsaedi, A., Hayat, T.: Exponential synchronization for a class of complex networks of networks with directed topology and time delay. Neurocomputing 266, 274-283 (2017)

12. Lee, S.H., Park, M.J., Kwon, O.M., Sakthivel, R.: Advanced sampled-data synchronization control for complex dynamical networks with coupling time-varying delays. Inf. Sci. 420, 454-465 (2017)

13. Xie, Q., Si, G., Zhang, Y., Yuan, Y., Yao, R.: Finite-time synchronization and identification of complex delayed networks with Markovian jumping parameters and stochastic perturbations. Chaos Solitons Fractals 86, 35-49 (2016)

14. Shi, L., Zhu, H., Zhong, S., Shi, K., Cheng, J.: Function projective synchronization of complex networks with asymmetric coupling via adaptive and pinning feedback control. ISA Trans. 65, 81-87 (2016)

15. Wang, J., Feng, J., Xu, C., Zhao, Y., Feng, J.: Pinning synchronization of nonlinearly coupled complex networks with time-varying delays using M-matrix strategies. Neurocomputing 177, 89-97 (2016)

16. Chen, W.-H., Jiang, Z., Lu, X., Luo, S.: Synchronization for complex dynamical networks with coupling delays using distributed impulsive control. Nonlinear Anal. Hybrid Syst. 17, 111-127 (2015)

17. Zhao, M., Zhang, H., Wang, Z., Liang, H.: Synchronization between two general complex networks with time-delay by adaptive periodically intermittent pinning control. Neurocomputing 144, 215-221 (2014)

18. Gong, W., Liang, J., Cao, J.: Matrix measure method for global exponential stability of complex-valued recurrent neural networks with time-varying delays. Neural Netw. 70, 81-89 (2015)

19. Song, Q., Zhao, Z., Liu, Y.: Impulsive effects on stability of discrete-time complex-valued neural networks with both discrete and distributed time-varying delays. Neurocomputing 168, 1044-1050 (2015)

20. Guo, X., Li, J.: A new synchronization algorithm for delayed complex dynamical networks via adaptive control approach. Commun. Nonlinear Sci. Numer. Simul. 17(11), 4395-4403 (2012)

21. Wei, R., Cao, J., Alsaedi, A.: Finite-time and fixed-time synchronization analysis of inertial memristive neural networks with time-varying delays. Cogn. Neurodyn. 12(1), 121-134 (2018)

22. Jing, T., Chen, F., Zhang, X.: Finite-time lag synchronization of time-varying delayed complex networks via periodically intermittent control and sliding mode control. Neurocomputing 199, 178-184 (2016)

23. Kaviarasan, B., Sakthivel, R., Lim, Y.: Synchronization of complex dynamical networks with uncertain inner coupling and successive delays based on passivity theory. Neurocomputing 186, 127-138 (2016)

24. Li, B.: Pinning adaptive hybrid synchronization of two general complex dynamical networks with mixed coupling. Appl. Math. Model. 40(4), 2983-2998 (2016)

25. Zhang, Z.-M., He, Y., Wu, M., Wang, Q.-G.: Exponential synchronization of chaotic neural networks with time-varying delay via intermittent output feedback approach. Appl. Math. Comput. 314, 121-132 (2017)

26. Wang, X., She, K., Zhong, S., Yang, H.: New result on synchronization of complex dynamical networks with time-varying coupling delay and sampled-data control. Neurocomputing 214, 508-515 (2016)

27. Shi, L., Zhu, H., Zhong, S., Zeng, Y., Cheng, J.: Synchronization for time-varying complex networks based on control. J. Comput. Appl. Math. 301, 178-187 (2016)

28. Hale, J.K., Verduyn Lunel, S.M.: Introduction to Functional Differential Equations. Springer, New York (1993)

29. Boyd, S., Ghaoui, L.E., Feron, E., Balakrishnan, V.: Linear Matrix Inequalities in System and Control Theory. SIAM, Philadelphia (1994)

30. Cai, S.M., He, Q.B., Hao, J.J., et al.: Exponential synchronization of complex networks with nonidentical time-delayed dynamical nodes. Phys. Lett. A 374, 2539-2550 (2010)

31. Wang, J.L., Qin, Z., Wu, H.N., et al.: Analysis and pinning control for output synchronization and $\mathrm{H} \infty$ output synchronization of multiweighted complex networks. IEEE Trans. Cybern. (2018). https://doi.org/10.1109/TCYB.2018.2799969

32. Ding, J., Cao, J., Feng, G., et al.: Exponential synchronization for a class of impulsive networks with time-delays based on single controller. Neurocomputing 218, 113-119 (2016)

33. Liu, Y., Guo, B.Z., Park, J.H., et al.: Nonfragile exponential synchronization of delayed complex dynamical networks with memory sampled-data control. IEEE Trans. Neural Netw. Learn. Syst. 29(1), 118-128 (2018)

34. Cheng, R., Peng, M., Yu, W.: Pinning synchronization of delayed complex dynamical networks with nonlinear coupling. Phys. A, Stat. Mech. Appl. 413(11), 426-431 (2014)

35. Fan, Y., Liu, H., Zhu, Y., et al.: Fast synchronization of complex dynamical networks with time-varying delay via periodically intermittent control. Neurocomputing 205(C), 182-194 (2016)

36. Cai, S., Hao, J., He, Q., et al.: Exponential synchronization of complex delayed dynamical networks via pinning periodically intermittent control. Phys. Lett. A 375(19), 1965-1971 (2011)

37. Su, H., Rong, Z., Wang, X., et al.: On decentralized adaptive pinning synchronization of complex dynamical networks. In: Proceedings of IEEE International Symposium on Circuits \& Systems, pp. 417-420 (2010) 